\title{
Preventing ischial pressure ulcers: II. Biomechanics
}

\author{
Hilton M. Kaplan ${ }^{\mathrm{a}, *}$, Lucinda L. Baker ${ }^{\mathrm{b}}$, Rahman Davoodi ${ }^{\mathrm{a}}$, Nga Ting Wong ${ }^{\mathrm{a}}$ and Gerald E. Loeb ${ }^{\mathrm{a}}$ \\ ${ }^{a}$ Department of Biomedical Engineering and Alfred Mann Institute for Biomedical Engineering, University \\ of Southern California, Los Angeles, CA, USA \\ ${ }^{\mathrm{b}}$ Department of Biokinesiology and Physical Therapy, University of Southern California, Los Angeles, CA, USA
}

\begin{abstract}
Background: Pressure ulcers (PUs) are common and debilitating wounds that arise when immobilized patients cannot shift their weight. Neuromuscular Electrical Stimulation (NMES) has been investigated for Pressure Ulcer Prevention (PUP) for over 20 years. Historically gluteus maximus (GM) has been considered an important actuator in attempting to redistribute seated pressures through NMES.

Methods: Analysis of skeletal biomechanics to quantify the value of GM relative to hamstring hip extensors (HS), using muscle moment models based on torques and rigid body mass estimates from the literature. Surface stimulation experiments $(n=10+1$, non-paralyzed) to validate model and identify promising stimulation sites and treatment strategies that would approximate healthy biomechanics.

Results: Literature values and Rigid Body Analysis estimate: $\sim 63 \mathrm{Nm}$ extensor torque requirement calculated for complete ipsilateral unloading of the buttocks. Muscle Moment Analysis: GM can provide 70\% of total hip extensor torque when walking vs. $18 \%$ when seated. HS can provide $100 \mathrm{Nm}$ hip extension torque when seated, exceeding $63 \mathrm{Nm}$ requirement. Surface Stimulation: ipsilateral seated interface pressure mean $-26 \%$ during HS stimulation vs. $+16 \%$ with GM; peak pressure area $-94 \%$ HS vs. $+213 \%$ GM.

Conclusions: GM activation reduces disuse atrophy and improves circulation, but appears neither required, nor desired, for unloading when seated. HS stimulation alone should be capable of sufficient unloading. This new proposed approach is explored clinically in companion paper III.
\end{abstract}

Keywords: Decubitus ulcer, neuromuscular electrical stimulation, pressure ulcer prevention, tissue biomechanics, spinal cord injury, gluteus maximus, hamstrings

\author{
Abbreviations \\ GM Gluteus Maximus \\ HS Hamstrings \\ NMES Neuromuscular Electrical Stimulation \\ PU Pressure Ulcer \\ PUP Pressure Ulcer Prevention \\ Quads Quadriceps \\ ROM Range of Motion \\ SCI Spinal Cord Injury
}

\footnotetext{
${ }^{*}$ Corresponding author: Hilton M. Kaplan, MD, PhD, PO Box 2337, Beverly Hills, CA 90213, USA. Tel.: +1 (310) 570 2822; Fax: +1 (310) 274 9931; E-mail hkaplan@alumni.usc.edu.
}

SIMM Software for Interactive Musculoskeletal Modeling (MusculoGraphics, Santa Rosa, CA)

\section{Introduction}

Pressure ulcers (PUs) are a debilitating pathology that can result in severe morbidity (e.g. sepsis, osteomyelitis, renal failure, cardiac failure) [27]. Ablebodied individuals do not get PUs because they can voluntarily contract their muscles, thereby shifting 
their weight while activating trophic mechanisms that maintain muscle bulk, strength and circulation. For over 2 decades there have been numerous clinical demonstrations of the value of neuromuscular electrical stimulation (NMES) for pressure ulcer prevention (PUP) [3-6, 9, 11, 19-24, 26, 28], but the actual physiological mechanisms involved remain less clear.

This is the second of three companion papers regarding the potential for chronic NMES to prevent ischial PUs in paraplegic patients. The first was a review of NMES for PUP. This paper presents a new theoretical and experimental analysis of the relevant musculoskeletal mechanics and considers previous attempts to use NMES in light of these findings. The final paper describes a pilot clinical study of a new technology that enables the strategy that is recommended on the basis of this analysis.

\section{Methods}

While it is recognized that spinal cord injured (SCI) subjects do not have the same proportions of their body segments and muscles as able-bodied individuals, ablebodied values were used in the analyses below for three reasons: 1) the aim was to design a treatment that ultimately will be implemented soon after injury for prophylaxis (before and to avoid atrophic changes); 2) a goal of the treatment is to strengthen and hypertrophy muscles to approach the bulk and strength of able-bodied counterparts; and 3) sufficient torque will be required to overcome, at worst, segment masses approaching those of able-bodied individuals.

\subsection{Estimation of extensor torque requirements}

A static rigid body analysis was conducted to get an estimate of the minimal muscle torques required to unload the ischium, and not to explore the kinematic consequences of those torques. It should be noted, therefore, that this section provides only estimates, but is included as background and for insight into the Muscle Moment Analyses that follow.

The rudimentary rigid body model was created to explore this behavior as follows: The body was represented by 8 rigid segments, seated in a rigid seat with footrest: 3 for each lower limb, 1 for the pelvis, and 1 for the torso, upper limbs, and head and neck: Fig. 1 demonstrates the requirements for complete unloading (MSC. visualNastran 4D; MSC Software, Santa Ana,
CA). The dimensions and mass of each segment were apportioned according to the HYBRID III Fiftieth Percentile Crash Test Dummy [25], which represents an average adult male of $1.75 \mathrm{~m}$ height and $78 \mathrm{~kg}$ mass $\quad(\operatorname{mBody}=$ mPelvis + mTorso $=9+46=55 \mathrm{~kg}$; $\mathrm{mLLimb}=\mathrm{mThigh}+\mathrm{mLeg}+\mathrm{mFoot}=6+4.75+$ $0.75=11.5 \mathrm{~kg}$ each). All segments were connected by passive joints, except for the R. hip (the site of "stimulation"), which was actuated with an extension torque motor. The pelvis-torso joint was considered rigid, on the assumption that for the small shifts we are modeling, the lumbo-sacral joint would offer only a limited range of motion (ROM) relative to the remaining joints. Similarly, in keeping with a paralyzed subject with neither voluntary nor reflexive activity of any muscles, the ankles and knees were modeled as frictionless, spherical joints; and the L. hip as a frictionless, revolute joint.

\subsection{Muscle moment analysis}

The relative hip extensor moments of the hamstring (HS) and gluteus maximus (GM) muscle groups were compared in light of the requirement for the minimal hip extension torque required to offload the ischium, as estimated above. An anatomically accurate model of muscle origin/insertion and wrapping was analyzed in SIMM (Software for Interactive Musculoskeletal Modeling; MusculoGraphics, Santa Rosa, CA) [7, 8] to identify the axis and magnitude of the relative hip torques that would be produced by each type of stimulation. All other muscles were inactivated and the musculoskeletal model was set with thighs neutral at $0^{\circ}$ of internal-external rotation and $0^{\circ}$ of abductionadduction. Force $(\mathrm{N})$ and moment arm $(\mathrm{m})$ data were derived for each contributory muscle across its full $\mathrm{ROM}$ in flexion-extension, to calculate the torque/ moment data for each muscle at maximal activation: Torque $(\mathrm{Nm})=$ Moment Arm $(\mathrm{m}) \times$ Force $(\mathrm{N})$. The moments of all muscles in each group were summed to plot GM total moment and HS total moment vs. ROM in flexion-extension (Fig. 2). Similar data were derived for internal-external rotation and abduction-adduction moments in the seated posture (Fig. 3).

\subsection{Surface stimulation validation}

To confirm the predictions of the models described above, we assessed the mechanical effects of the muscles on seated posture and pressure distributions by reanalyzing surface stimulation data from 10 healthy 

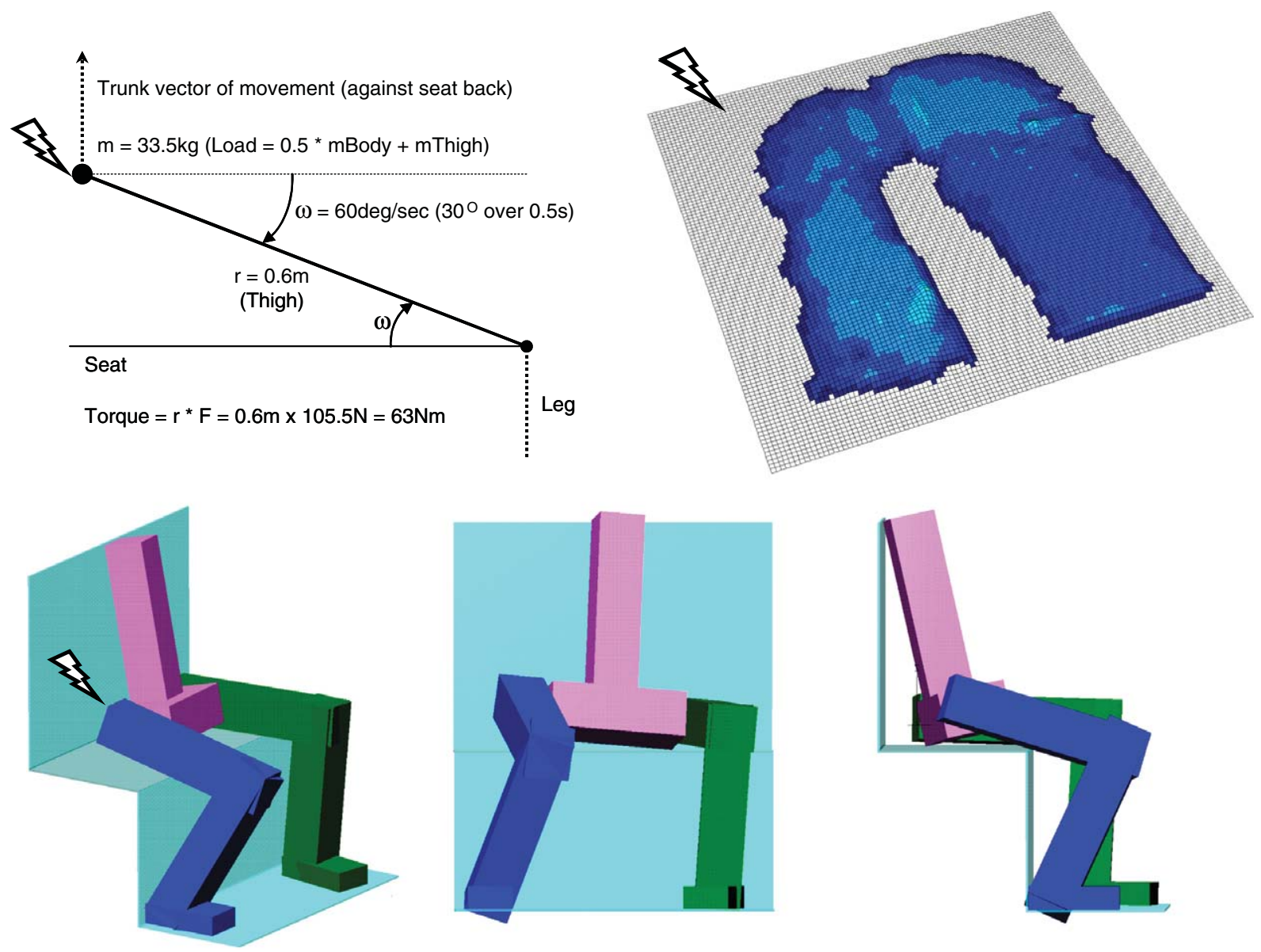

Fig. 1. Rigid body analysis of R. hip extension torque, for extreme unloading. Note unloading under ipsilateral buttock (and contralateral buttock to a lesser degree). This simulates well the experimental findings from surface stimulation interface maps (inset right and Fig. 6).

subjects collected previously by our group [1]. A seat cushion with an $8 \times 8$ grid of air-filled cells had been used to measure seated interface pressures both at rest, and during stimulation, of different muscle configurations and voluntary shifting. Surface NMES used a symmetrical biphasic waveform with a frequency of $35 \mathrm{pps}$ and a $250 \mu \mathrm{s}$ phase duration. Tests were randomized, and each was run 3 times to determine average rest and stimulation values. The muscle combinations assessed were: (1) Quadriceps (Quads) + GM; (2) Quads + gluteus medius; (3) HS + GM; (4) GM; (5) Quads; and (6) voluntary weight-shifting. The authors had noted that HS + GM stimulation was statistically no different from voluntary weight-shifting (in terms of mean seating pressures, normalized in proportion to body weight, using a Tukey Test).
These observations of Avruskin, et al. [1] were explored further, and extended to higher spatial resolution, in a more recent experiment on a healthy volunteer (presented in abstract form in Kaplan [15]). Surface stimulation of the hip extensors was performed in a single healthy male subject $(39 \mathrm{y} / \mathrm{o}, 76 \mathrm{~kg}$, $1.73 \mathrm{~m})$. Oval electrodes $(3.8 \mathrm{~cm} \times 6.4 \mathrm{~cm}$ Gentle Stim R Plus; Medical Devices Intl, Saint Louis, MO) were placed over each muscle's bulk with long axes perpendicular to the fibers, $3.8 \mathrm{~cm}$ apart. The subject was seated in a wheelchair with foot and arm rests: thighs flat; hips, knees, ankles and elbows flexed to $90^{\circ}$; calves restrained posteriorly. Interface pressures were measured at $10 \mathrm{~Hz}$ using a $45.7 \mathrm{~cm} \times 45.7 \mathrm{~cm}$ array of sensors (X36 System; XSensor Technology, Calgary, AB, Canada) on a $10.2 \mathrm{~cm}$ thick high-density 


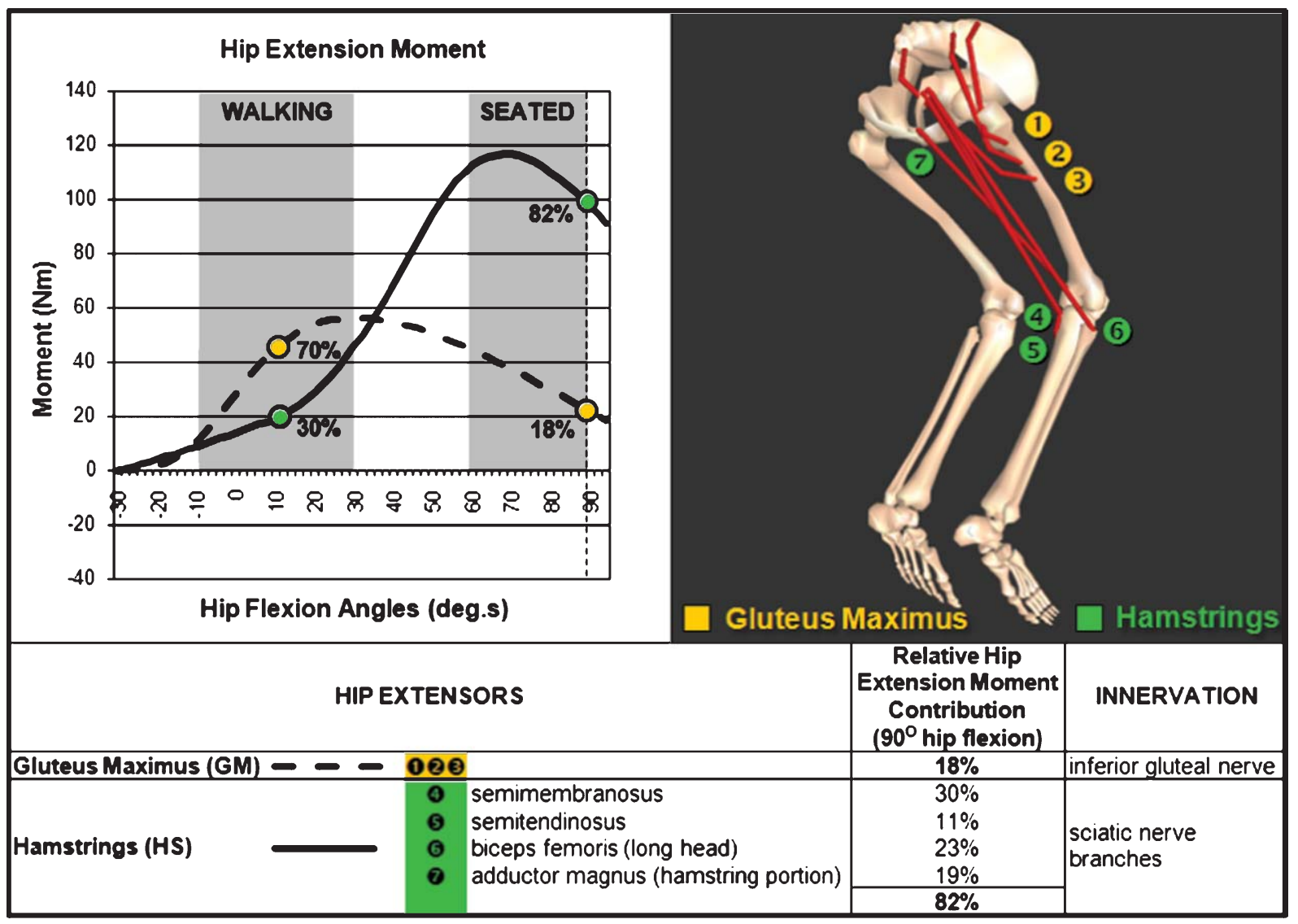

Fig. 2. Analysis of the relative contributions of the hip extensors to the total hip extension moment.
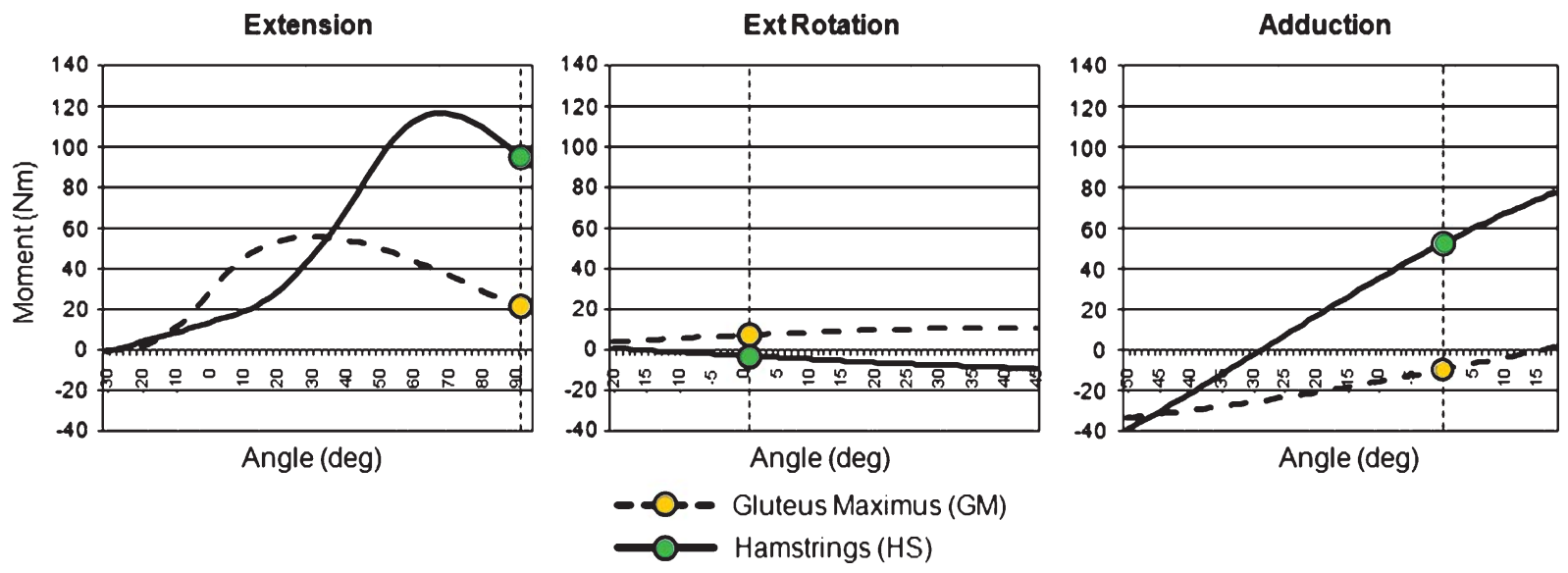

Fig. 3. Comparing extension, external rotation and adduction moments for GM stimulation and HS stimulation: GM moments (circles on dashed lines) are all of a similar order of magnitude at the angles of interest for neutral, seated posture (extension $21.7 \mathrm{Nm}$; external rotation $7.1 \mathrm{Nm}$; abduction $9.9 \mathrm{Nm}$ ). For HS moments (circles on solid lines), however, the extension torque $(97.3 \mathrm{Nm})$ dominates that for external rotation $(-2.6 \mathrm{Nm})$. The moderate adduction component $(52.2 \mathrm{Nm})$ is of less concern as it will be stopped in the midline by the contralateral limb or a commercial spacer if needed. 
foam cushion $\left(45 \mathrm{~kg} \cdot \mathrm{m}^{-3}\right)$, on top of a flat hard board. The XSensor system is a capacitive array of $1,296 \times 1.3 \times 1.3 \mathrm{~cm}^{2}$ sensing elements (accuracy $= \pm 1.3 \mathrm{kPa} \quad(\sim 10 \mathrm{mmHg})$, range $=1.3-26.7 \mathrm{kPa}$ $(\sim 10-200 \mathrm{mmHg}))$. NMES consisted of $10 \mathrm{~s}$ trains at 35 pps interspersed with $10 \mathrm{~s}$ pauses (FastStart EMS; Vision Quest, Irvine, CA). Amplitude of biphasic pulses (300 $\mu$ s duration) varied between $60-100 \mathrm{~mA}$ to control recruitment, aiming for tetany / maximal unloading by the appropriate muscle groups. Seating pressure distributions were recorded and averaged from 3 runs each of GM, HS and Quads stimulation, individually and in all combinations.

While interface pressure mapping is commonly used to assess NMES for PUP, an important drawback has been that the variations in peak pressure have usually been reported for only very small areas immediately beneath the ischial tuberosities (e.g. a single cell of $1.3 \times 1.3=1.6 \mathrm{~cm}^{2}[23,24]$, or a zone of $3.6 \times 3.6=13 \mathrm{~cm}^{2}[11,28]$; see review in companion paper I [14]). However large areas are at risk if the closed capillaries are not able to reperfuse through adequate unloading, and the ability to recover from ischemic damage may depend on mass effects. To address these concerns, two indices of seating pressure were defined and extracted from the combined data using Matlab ${ }^{\mathrm{TM}}$ (The MathWorks, Natick, MA). The first is a relative indicator of large area improvements; the second is an absolute indicator of actual anatomical regions where insufficient unloading is likely to persist despite stimulation (Fig. 4).

\subsubsection{MPZs, mean pressure zones}

The MPZs were 4 large user-defined areas, one within each quadrant of the seated surface, for the comparison of average pressure at rest and during stimulation. The 4 quadrants were defined by a coronal plane through the inferior gluteal creases and the perpendicular midline, as the R. and L. buttock quadrants, and R. and L. thigh quadrants. Each MPZ was set the same size and shape in each quadrant, their size being normalized to $80 \%$ of the resting contact surface beneath the ipsilateral ischium (i.e. on the side of stimulation). This was facilitated by dividing each of the actual pressure cells mapped into higher resolution cells of that same pressure (for the low resolution maps: $8 \times 8$ cells $\times 45$ each $=360 \times 360$; for the high resolution maps: $36 \times 36$ cells $\times 10$ each $=360 \times 360$ ).
The buttock quadrants were centered under the ischiae, while the thigh quadrants were placed symmetrically at the most distal edges of measured thigh contact. The average pressure in these zones was compared before and after stimulation to derive a relative performance index (\% change) to compare results between subjects and over time.

\subsubsection{PPA, peak pressure area}

The PPA was the area of cells with pressures $\geq 8 \mathrm{kPa}$ $(60 \mathrm{mmHg}$ ) beneath the ipsilateral buttock. This value was chosen based on finite element model estimates presented elsewhere and beyond which we believe capillaries within the deep tissues could be occluded [15]. A precise cut-off value, however, cannot be defined as: 1) much dispute persists still to this day with regard to capillary closing pressures (following the seminal work of Landis [18] and Kosiak [16, 17], and as reviewed by Goossens [10]); 2) the effects of shear on capillary patency (discussed in companion paper I [14]) were not modeled; and 3) the relationships between surface pressures and internal compressive stresses are complex and non-linear. At the least, the value offers a benchmark for systematic comparison of the area that may remain unrelieved by stimulation and so be at increased risk of Deep Tissue Injury (DTI). The PPAs' size (area) and position (\% overlap and centroid shift [2]) were compared at rest and during stimulation.

\section{Results}

\subsection{Estimation of extensor torque requirements}

While recognizing the rudimentary and exploratory nature of this portion of our analysis, the torque requirement to completely unload the ipsilateral pelvis was calculated to be $63 \mathrm{Nm}$. This result is consistent with the $\sim 80 \mathrm{Nm}$ found by Waters, et al. [29] in experiments on normal subjects in which maximal hip extension was measured with and without lidocaine block of the sciatic nerve distal to the inferior gluteal nerve innervating GM. Qualitatively the model behaved as expected (Fig. 1). It suggested that unilateral hip extension could afford adequate pressure relief ipsilaterally, and may provide some more minor relief contralaterally too. 
(a)

Good Result

(HS alone)

(b)

Intermediate Result

(HS+GM+Quads)

(c)

Poor Result

(GM alone)
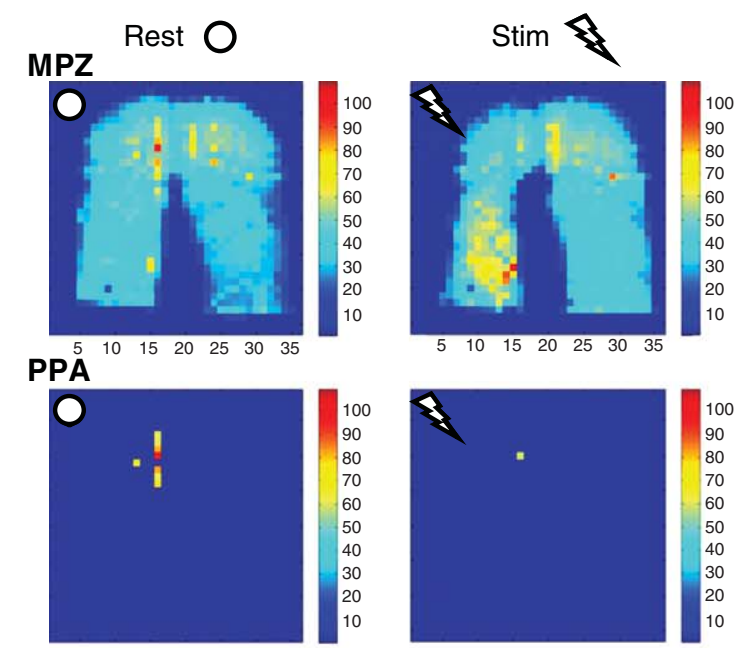

MPZ

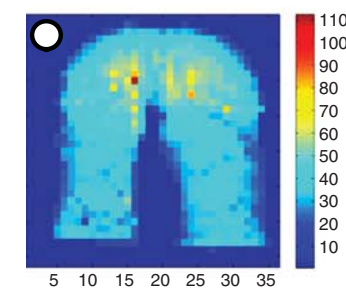

PPA
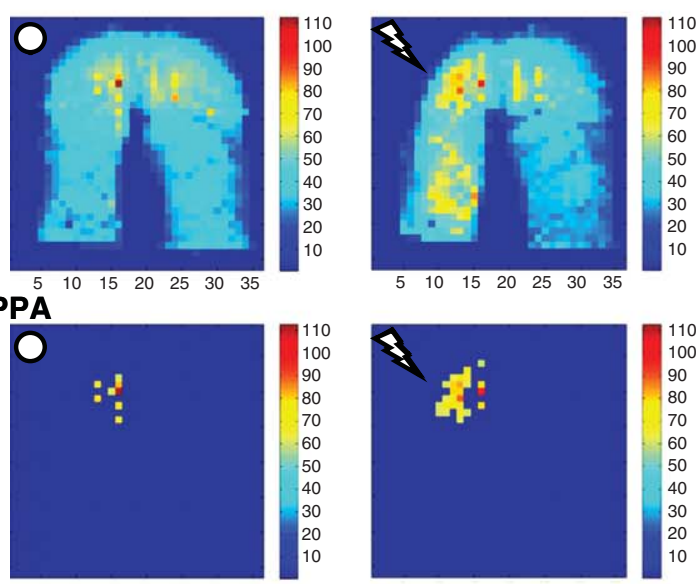

MPZ

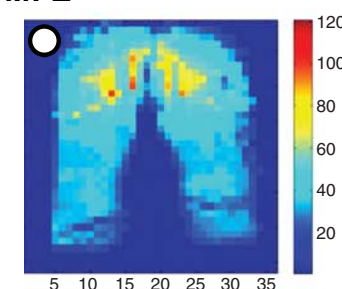

PPA
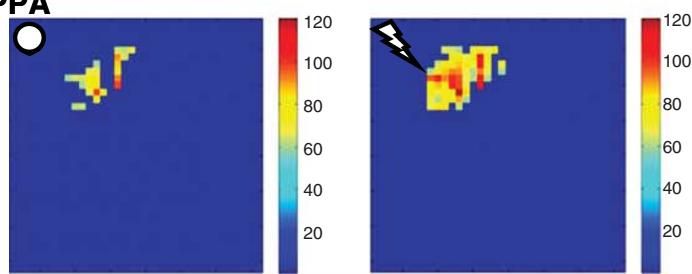

Difference \%
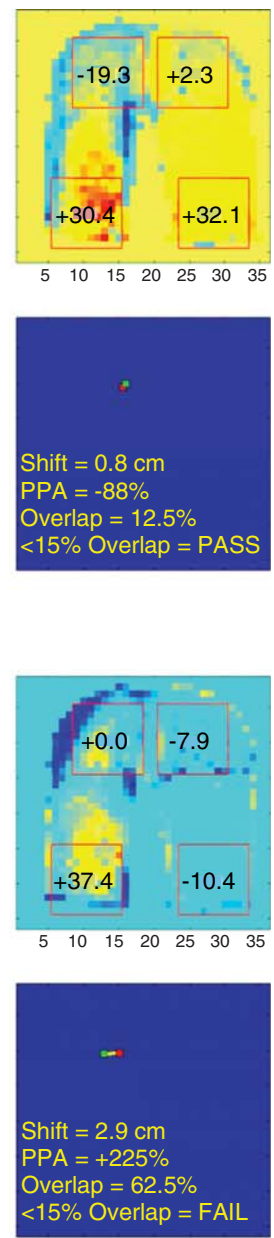

$<15 \%$ Overlap = FAIL
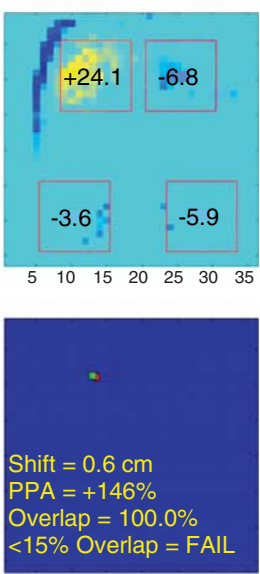

Fig. 4. (Continued) 


\subsection{Muscle moment analysis}

\subsubsection{Total hip extensor torque (Fig. 2)}

Comparing the GM and HS component sums of hip extensor torque, confirmed that GM was the dominant hip extensor (providing 70\%) when walking, i.e. between heel contact and toe-off of a regular gait cycle (hip angle $+30^{\circ}$ to $-10^{\circ}$ ) [12]. When seated at $90^{\circ}$ of hip flexion, however, $82 \%$ of the available extensor torque originated from HS; and this proportion persisted over $\sim 20^{\circ}$ of hip extension/unloading. Whereas it was estimated that approximately $\sim 60 \mathrm{Nm}$ of extension torque would be required for complete isometric unloading (above), the contribution from HS stimulation alone provided $\sim 100 \mathrm{Nm}$; while GM stimulation provided only $\sim 20 \mathrm{Nm}$, when seated. This suggests that even a non-atrophied GM would be insufficient to achieve ischial elevation, while a non-atrophied HS alone, would easily suffice. These results are consistent with the $\sim 80 \mathrm{Nm}$ of HS torque calculated by Waters, et al. [29].

\subsubsection{Comparison of extension, rotation and abd-adduction moments (Fig. 3)}

For maximal GM stimulation in a seated posture, the moments in abduction $(9.9 \mathrm{Nm})$ and external rotation $(7.1 \mathrm{Nm})$ were predicted to be substantial when compared to the extensor moment of $21.7 \mathrm{Nm}$ (46\% and $33 \%$ respectively). Therefore with GM actuation alone, not only is extension weak in the seated posture (above), but also relatively substantial effects could be anticipated to result from all 3 of these moments compared to the desired pure hip extension.

HS stimulation offered a much larger extensor moment $(97.3 \mathrm{Nm})$, with only a small degree of internal rotation $(2.6 \mathrm{Nm})$. The predicted adduction component was moderately high $(52.2 \mathrm{Nm})$, but adduction is of less clinical significance than the other moments as it is stopped in the midline by the contralateral limb, or by employing a commercially available knee spacer if necessary. The full pattern of these torques, obtained in all three axes for each of the muscle groups, was applied to the musculoskeletal mechanics model to get a subjective assessment of their effects (Fig. 5). These rigid body simulations support that $\mathrm{HS}$ activation would result in ischial elevation, while GM activation could not, and would instead cause lateral sliding of the thigh (which could tend to increase shear).

\subsection{Seating pressures}

Figure 4 shows results from the recent additional subject for HS stimulation alone (upper panel), for $\mathrm{HS}+\mathrm{GM}+\mathrm{Quads}$ stimulation combined (middle panel), and for GM stimulation alone (lower panel), demonstrating a good, an intermediate, and a poor result, respectively. In these examples average pressure (MPZ) beneath the ipsilateral buttock decreased $19 \%$ during stimulation of HS (upper panel). Combining GM and Quad with HS stimulation actually increased this MPZ by $5 \%$ (middle panel). GM stimulation alone produced a $24 \%$ increase in MPZ pressure (lower panel). HS stimulation alone produced an $88 \%$ reduction in PPA size and in PPA overlap, with only one cell remaining unrelieved through both rest and stimulation phases (i.e. still $\geq 8 \mathrm{kPa}(60 \mathrm{mmHg}))$ in the upper panel. The middle and lower panels demonstrate progressively worsening results, with the remaining PPA size and \% overlap progressively increasing, and the centroid shift progressively decreasing.

While the authors recognize the limitations of combining quantitative data from different studies with different dimensions/resolutions of the sensor cells, the results of both studies were very similar and so the addition of a single subject at a higher resolution was considered acceptable in this circumstance.

Fig. 4. Example runs of surface stimulation data. Panel (a): HS stimulation alone; Panel (b): HS + GM + Quads stimulation combined; Panel (c): GM stimulation alone. These examples demonstrate a good, an intermediate, and a poor result, respectively. In each panel the top row shows the raw data (accuracy $\pm 1.3 \mathrm{kPa}(10 \mathrm{mmHg})$ ), and the bottom row shows only cells with pressures of $\geq 8 \mathrm{kPa}(60 \mathrm{mmHg})$. In each case rest data is shown in the first column, stimulation data in the second column, and the difference between rest and stimulation in the third column (Matlab ${ }^{\mathrm{TM}}$ ). Note particularly the differences in pressure and in contact surface area, on the stimulated sides during stimulation vs. rest. $M P Z s=$ Mean Pressure Zones (R. upper image in each panel): User-defined areas in each quadrant in which we compare the average pressure at rest and during stimulation. They are the same size and shape in all 4 quadrants (see text). These examples demonstrate a $19 \%$ decrease in average pressure beneath the ipsilateral buttock during stimulation of HS (Panel (a)), but a 5\% increase when HS stimulation is combined with GM and quadriceps (Panel (b)), and a 24\% increase with GM stimulation alone (Panel (c)). Weight shifts to the thighs indicate successful unloading. $P P A=$ Peak Pressure Area (lower images in each panel): Area of cells with pressures of $\geq 8 \mathrm{kPa}(60 \mathrm{mmHg})$ beneath the ipsilateral buttock. These are areas in which we believe capillaries within the deep tissues are likely to remain occluded and at risk of DTI (see text). The PPA's size (area) and position (\% overlap and centroid shift) are compared at rest and during stimulation. 
(a)

\section{GM Activation}

(b)

\section{HS Activation}

Extension

Int rotation

Adduction
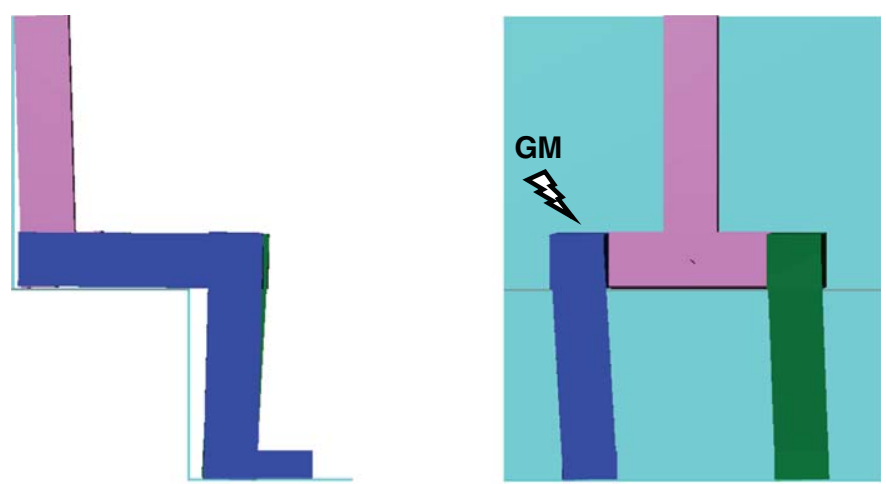

$1.7 \mathrm{Nm}$

$7.1 \mathrm{Nm}$

$9.9 \mathrm{Nm}$

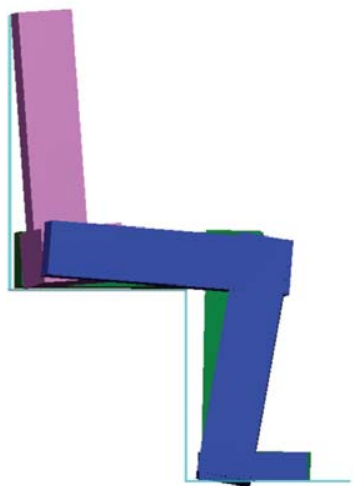

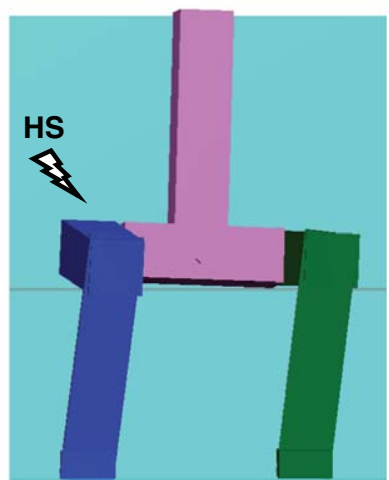

Fig. 5. Rigid body simulations of activating GM (upper panel) and HS (lower panel) using full pattern of torques in all three axes (from Fig. 3); shown to maximal "unloading" for GM (reached at $1.5 \mathrm{~s}$ ) and successful unloading for HS (reached at $0.2 \mathrm{~s}$ ). Note: with HS activation the position of the center of mass does not shift forward significantly, as although there is a shift of pressure towards the thighs, this is countered as the hip extends the torso towards the seatback. This is seen (in the extreme) here. Absolute values provide gross estimates only (due to model limitations), but their relative magnitudes support important differential effects: HS activation results in ischial elevation; GM activation causes lateral sliding of thigh without elevation, which could tend to increase shear.

Combined MPZ data from all subjects $(n=10+1)$ (Fig. 6) demonstrated that HS stimulation produced a significant reduction in seated interface pressures ipsilaterally (mean $-26 \%$; from mean $5.7 \pm 1.0 \mathrm{kPa}(43 \pm$ $7.6 \mathrm{mmHg})$ at rest to $4.3 \pm 0.9 \mathrm{kPa}(32 \pm 6.8 \mathrm{mmHg})$ during stimulation), as was predicted by the model; together with a large reduction in total contact area (mean $-25 \%$; from mean $341.5 \pm 17.6 \mathrm{~cm}^{2}$ at rest to $257.9 \pm 13.5 \mathrm{~cm}^{2}$ during stimulation). GM stimulation on the other hand increased the recorded seating pressures ipsilaterally (mean $+16 \%$; from mean $5.5 \pm$ $0.9 \mathrm{kPa}(41 \pm 6.5 \mathrm{mmHg})$ at rest to $6.4 \pm 1.1 \mathrm{kPa}(48 \pm$ $8.1 \mathrm{mmHg}$ ) during stimulation), with only a small reduction in contact surface area (mean $-10 \%$; from mean $346.0 \pm 13.8 \mathrm{~cm}^{2}$ at rest to $313.1 \pm 21.7 \mathrm{~cm}^{2}$ during stimulation). The distribution of pressures shown in Fig. 6 seems to suggest that the bulging of the stimulated GM muscle served to concentrate seating pressure in a denser region ipsilaterally, even slightly off-loading the contralateral buttock (mean $-4 \%$ ). Conversely, HS stimulation reduced seating pressures both ipsilaterally (mean $-26 \%$ as above) and contralaterally (mean $-8 \%$ ), with pressure shifts to the distal thighs (seen also in Fig. 4 upper \& middle panels). This represents effective unloading on the stimulated side, presumably elevating the pelvis in total (albeit more effectively on the side of stimulation). Irrespective of the torques generated at the hip, the weight of the torso remains unchanged: only its distribution over different portions of the seating surface can change. Such weight shifts to the thighs are therefore indicative of successful unloading at the buttocks. They are of lit- 


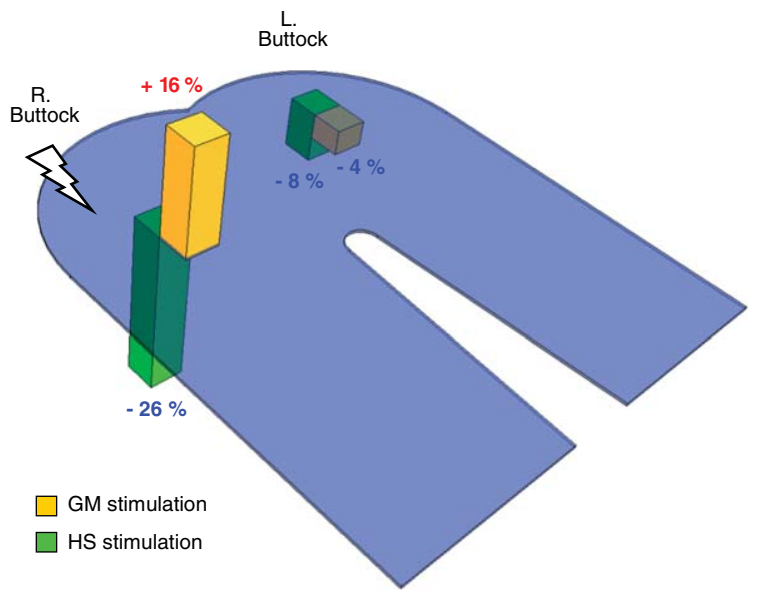

Fig. 6. Percentage pressure change in MPZs during surface stimulation $(n=10+1$, see text): HS stimulation benefits both sides significantly more than GM stimulation, and especially so ipsilaterally (where GM stimulation aggravates interface pressures).

tle concern in themselves because these areas have no boney prominences and are not at risk for PUs (they are also the normal areas to which mobile individuals unload when shifting weight).

Combined PPA data were compared at rest and during stimulation, in terms of size and position. (1) Size: HS stimulation resulted in almost complete elimination of PPA (mean $-94 \%$; from mean $25.8 \pm 5.9 \mathrm{~cm}^{2}$ at rest to $1.6 \pm 0.8 \mathrm{~cm}^{2}$ during stimulation); GM stimulation, on the other hand, resulted in over a trebling of PPA (mean $+213 \%$; from mean $25.8 \pm 7.3 \mathrm{~cm}^{2}$ at rest to $80.6 \pm 12.4 \mathrm{~cm}^{2}$ during stimulation), and with none of the at-rest PPA relieved during GM stimulation. (2) Position: The PPA centroid shifts with HS and GM stimulation were similar (mean $1.3 \mathrm{~cm}$ and $1.1 \mathrm{~cm}$ respectively), however the clinically predictive value of this measure is questionable here due to the large differences in absolute PPA size that were seen.

Finally, it was also noted anecdotally that with the hip flexed at $90^{\circ}$ the greatest effect of GM stimulation alone, at a 3+clinical muscle strength, was a modest external rotation and abduction of the thigh rather than strong hip extension, in keeping with the model predictions.

\section{Discussion}

Rigid body analysis was employed to confirm literature estimates of the minimal muscle torques required to unload only, and not to explore the kinematic consequences of these. A particularly beneficial avenue for future work, however, would be a more complete rigid body analysis to plot external forces and motion as a function of hip torque. An understanding of these external forces would provide insight into possible wheelchair modifications and/or other muscle stimulation sites, that may assist or improve the system, as well as adverse effects that may occur with chronic use (such as excessive shearing and/or skin abrasion at locations such as the thighs). Such an analysis would allow for a more accurate representation of the mechanisms of unloading that are being evaluated here. Changing the model assumptions and constraints could then be used to estimate the effects of variations in the proposed treatment protocol such as repositioning of foot supports and the addition of lower limb restraints.

The results of both the modeling simulations and the surface stimulation experiments suggest that although GM is the dominant hip extensor during walking, even tetanic activation of a healthy GM is unlikely to provide adequate hip extension to elevate the ischium and reduce seated pressures sufficiently to avoid ischemic damage to soft tissues. The question then arises as to what is actually accounting for the favorable results reported clinically with GM stimulation alone [3-6].

NMES of the buttock muscles is likely to be valuable in terms of its trophic effects, improving vascularity and soft tissue bulk. However, in order to prevent ischemic damage to these tissues and consequent PU formation while seated, it would seem necessary to reduce pressures (compressive stresses) periodically; in all contact areas and to below those pressures that occlude blood flow in capillary beds. Because the weight of the body that must be supported is constant, NMES will be effective in meeting these requirements only if it produces regular and substantial shifts in the distribution of seating pressures between the buttocks and the thighs.

The analysis presented here suggests that two separate muscle groups, GM and HS, need to be stimulated selectively, at different times, to take advantage of the separate therapeutic mechanisms detailed above: $\mathrm{GM}+\mathrm{HS}$ for conditioning of the buttock tissues while non-weightbearing, and HS alone for load relief while seated. This new proposed approach to PU prevention is explored clinically in companion paper III [13]. The parameters used in that study were adapted from those defined here, and include: 
1. Pavg (Average Pressure $)=$ the average pressure in the contact area of each quadrant; normalized to the average pressure across the full contact area to facilitate intersubject comparisons (this is a version of MPZ which we believed would be easier to implement in the clinical setting while providing similar value).

2. $P \max ($ Maximum Pressure $)=$ the maximum pressure in each quadrant; normalized to the average pressure across the full contact area to facilitate intersubject comparisons.

3. PPA (Peak Pressure Area) $=$ the area in each quadrant with pressures $\geq 8 \mathrm{kPa}(60 \mathrm{mmHg})$, as an indicator of likely capillary occlusion; normalized to the overall contact area across all 4 quadrants to facilitate intersubject comparisons.

\section{Conclusions}

This analysis suggests that while GM activation reduces disuse atrophy and improves circulation, it appears to be neither required, nor desired, for unloading when seated. HS stimulation alone should be capable of sufficient unloading. Therefore these two separate muscle groups need to be stimulated selectively, at different times, to take advantage of their separate therapeutic mechanisms: GM+HS for conditioning of the buttock tissues while non-weightbearing, and HS alone for load relief while seated. Load relief must be accomplished regularly throughout the day by patients or their caregivers while minimizing any added risks of the intervention itself. In the final companion paper III (13) we describe a pilot study of a novel technology that can meet these requirements.

\section{Acknowledgement}

This research was funded by the Alfred Mann Institute at the University of Southern California and the National Science Foundation Engineering Research Center for Biomimetic MicroElectronic Systems.

\section{References}

[1] A. Avruskin and T. McCauley, The Effectiveness of NMES in producing involuntary weight shifting in normal subjects, Masters Thesis Department of Biokinesiology and Physical Therapy, University of Southern California, Los Angeles, CA. 1992.
[2] F.P. Beer, E.R. Johnston and J.T. DeWolf, Mechanics of Materials 3rd Ed, McGraw Hill, New York, 2001.

[3] K.M. Bogie, S.I. Reger, S.P. Levine and V. Sahgal, Electrical stimulation for pressure sore prevention and wound healing, Asst Technol 12 (2000), 50-66.

[4] K.M. Bogie, RJ. Triolo and J. Chae, Improving the health of paralyzed tissue using electrical stimulation, Proc 3rd Conf Rehab Res Dev, US Dept of Veterans Affairs, Feb 2002.

[5] K.M. Bogie and R.J. Triolo, Effects of regular use of neuromuscular electrical stimulation on tissue health, J Rehab Res Dev 40(6) (Nov-Dec 2003), 469-476.

[6] K.M. Bogie, X. Wang and R.J. Triolo, Long-term prevention of pressure ulcers in high-risk patients: a single case study of the use of gluteal neuromuscular electric stimulation, Arch Phys Med Rehabil 87 (2006), 585-591.

[7] S.L. Delp, Lower Limb Model for SIMM (Software for Interactive Musculoskeletal Modeling), MusculoGraphics Inc, Santa Rosa, CA, Jan 2007.

[8] S.L. Delp and J.P. Loan, A computational framework for simulating and analyzing human and animal movement, Computing in Science \& Eng 5 (Sep-Oct 2000), 46-55.

[9] A.C. Ferguson, J.F. Keating, M.A. Delargy and B.J. Andrews, Reduction of seating pressure using FES in patients with spinal cord injury, A preliminary Report. Paraplegia 30(7) (Jul 1992), 474-478.

[10] R.H. Goossens, A short history of progression of research into seating and postural support, Disabil Rehabil Assist Technol 2(4) (Jul 2007), 249-254.

[11] T.W.J. Janssen, A. van Londen, M. Herwegh, C.H. van der Zee, C.A.J. Smit, A. Niezen and A. Daffertshofer, Surface electrical stimulation of gluteal muscles reduces sitting pressure in wheelchair users with a spinal cord injury, Proc 12th International Functional Electrical Stimulation Soc Philadelphia, PA, Nov 2007.

[12] M.P. Kadaba, H.K. Ramakrishnan and M.E. Wootten, Measurement of lower extremity kinematics during level walking, J Orthop Res 8(3) (May 1990), 383-392.

[13] H.M.Kaplan, L.L. Baker, S. Rubayi and G.E.Loeb, Preventing ischial pressure ulcers: III. Clinical Pilot Study of Chronic Neuromuscular Electrical Stimulation Companion Paper, Apr 2011 a.

[14] H.M. Kaplan and G.E. Loeb, Preventing ischial pressure ulcers: I. Review of Neuromuscular Electrical Stimulation, Companion Paper, Apr 2011b.

[15] H.M. Kaplan, G.E. Loeb, L.L. Baker and R. Davoodi, BION active seating for pressure ulcer prevention: surface stimulation and finite element modeling for planning treatments, Proc 11th International Functional Electrical Stimulation Soc, Sendai, Japan, Sep 2006.

[16] M. Kosiak, Etiology and pathology of ischemic ulcers, Arch Phys Med Rehabil 40(2) (Feb 1959), 62-69.

[17] M. Kosiak, Etiology of decubitus ulcers, Arch Phys Med Rehabil 42 (Jan 1961), 19-29.

[18] E.M. Landis, Micro-injection studies of capillary blood pressure in human skin, Heart 15 (1930), 209-228.

[19] S.P.Levine, Functional electrical stimulation for pressure sore inhibition. USPTO Patent 4,727,878. Assignee: University of Michigan, Ann Arbor, MI. Filed: Sep 1985. Issued: Mar 1988

[20] S.P. Levine, R.L. Kett, B.S. Cederna, L.D. Bowers and S.V. Brooks, Electrical muscle stimulation for pressure variation at the seating interface, J Rehab Res Dev 26(4) (1989), 1-7.

[21] S.P. Levine, R.L. Kett, P.S. Cederna and S.V. Brooks, Electric muscle stimulation for pressure sore prevention: tissue 
shape variation, Arch Phys Med Rehabil 71(3) (Mar 1990a), 210-215.

[22] S.P. Levine, R.L. Kett, M.D. Gross, B.A. Wilson, P.S. Cederna and J.E. Juni, Blood flow in the gluteus maximus of seated individuals during electrical muscle stimulation, Arch Phys Med Rehabil 71(9) (Aug 1990b), 682-686.

[23] L.Q. Liu, G.P. Nicholson, S.L. Knight, R. Chelvarajah, A. Gall, F.R. Middleton, M.W. Ferguson-Pell and M.D. Craggs, Pressure changes under the ischial tuberosities of seated individuals during sacral nerve root stimulation, J Rehabil Res Dev 43(2) (Mar-Apr 2006a), 209-218.

[24] L.Q. Liu, G.P. Nicholson, S.L. Knight, R. Chelvarajah, A. Gall, F.R.I. Middleton, M.W. Ferguson-Pell and M.D. Craggs, Interface pressure and cutaneous hemoglobin and oxygenation changes under ischial tuberosities during sacral nerve root stimulation in spinal cord injury, J Rehabil Res Dev 43(4) (Jul-Aug 2006b), 553-564.

[25] National Highway Traffic Safety Administration, HYBRID III fiftieth percentile crash test dummy. Vehicle Research
\& Test Center, Pedestrian \& Applied Biomechanics Div. Jan 2007.

[26] L.R. Solis, D.P. Hallihan, R.R.E. Uwiera, R.B. Thompson, E.D. Pehowich and V.K. Mushahwar, Prevention of pressure-induced deep tissue injury using intermittent electrical stimulation, J Appl Physiol 102 (Feb 2007), 1992-2001.

[27] D.R.Thomas, Prevention and treatment of pressure ulcers: What works? What doesn't? Cleve Clin J Med 68(8) (Aug 2001),704-707,710-714,717-722.

[28] A. van Londen, M. Herwegh, C.H. van der Zee, A. Daffertshofer, C.A. Smit, A. Niezen and T.W. Janssen, The effect of surface electric stimulation of the gluteal muscles on the interface pressure in seated people with spinal cord injury, Arch Phys Med Rehabil 89(9) (Sep 2008), 1724-1732.

[29] R.L. Waters, J. Perry, J.M. McDaniels and K. House, The relative strength of the hamstrings during hip extension, $J$ Bone Joint Surg Am 56 (1974), 1592-1597. 

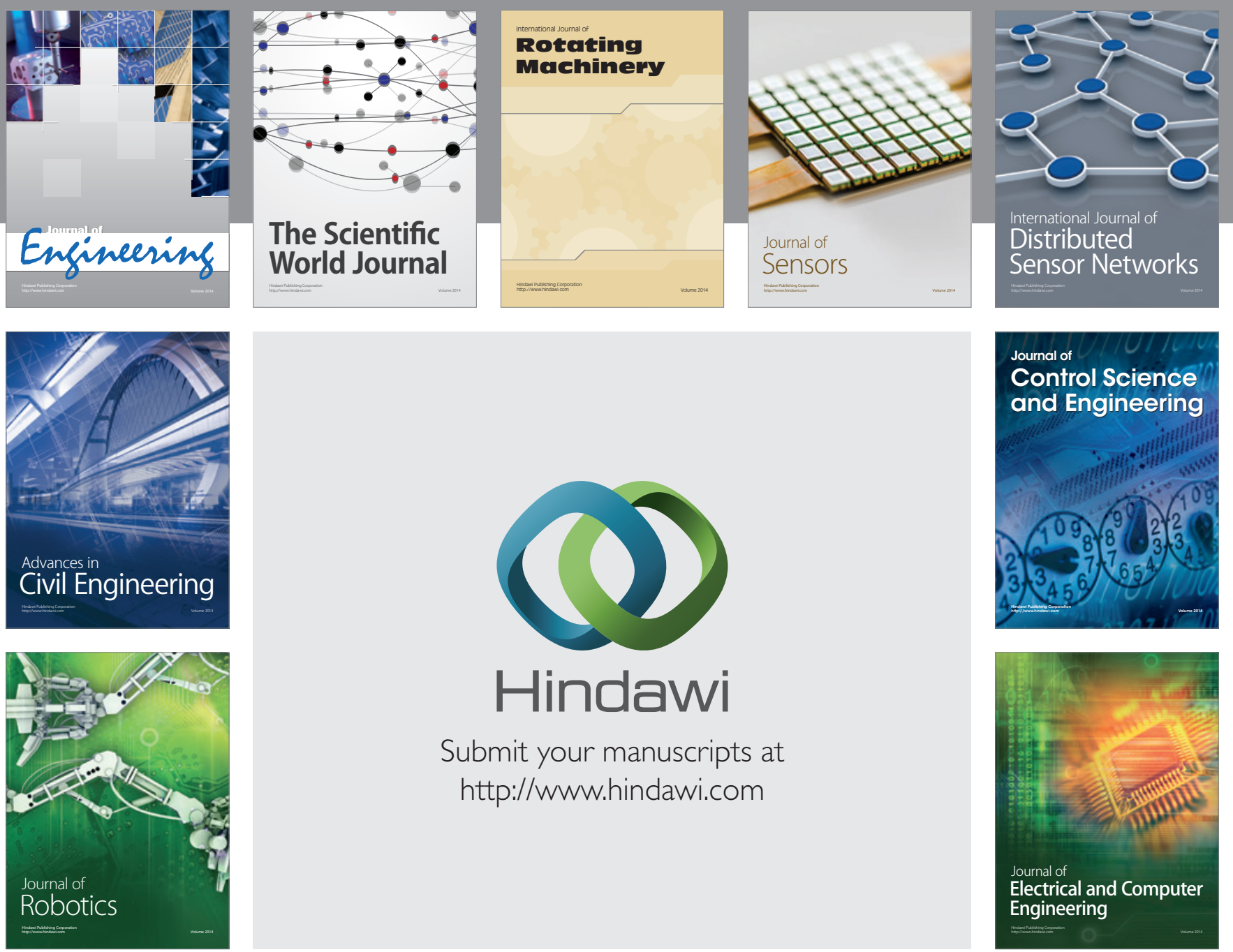

Submit your manuscripts at

http://www.hindawi.com
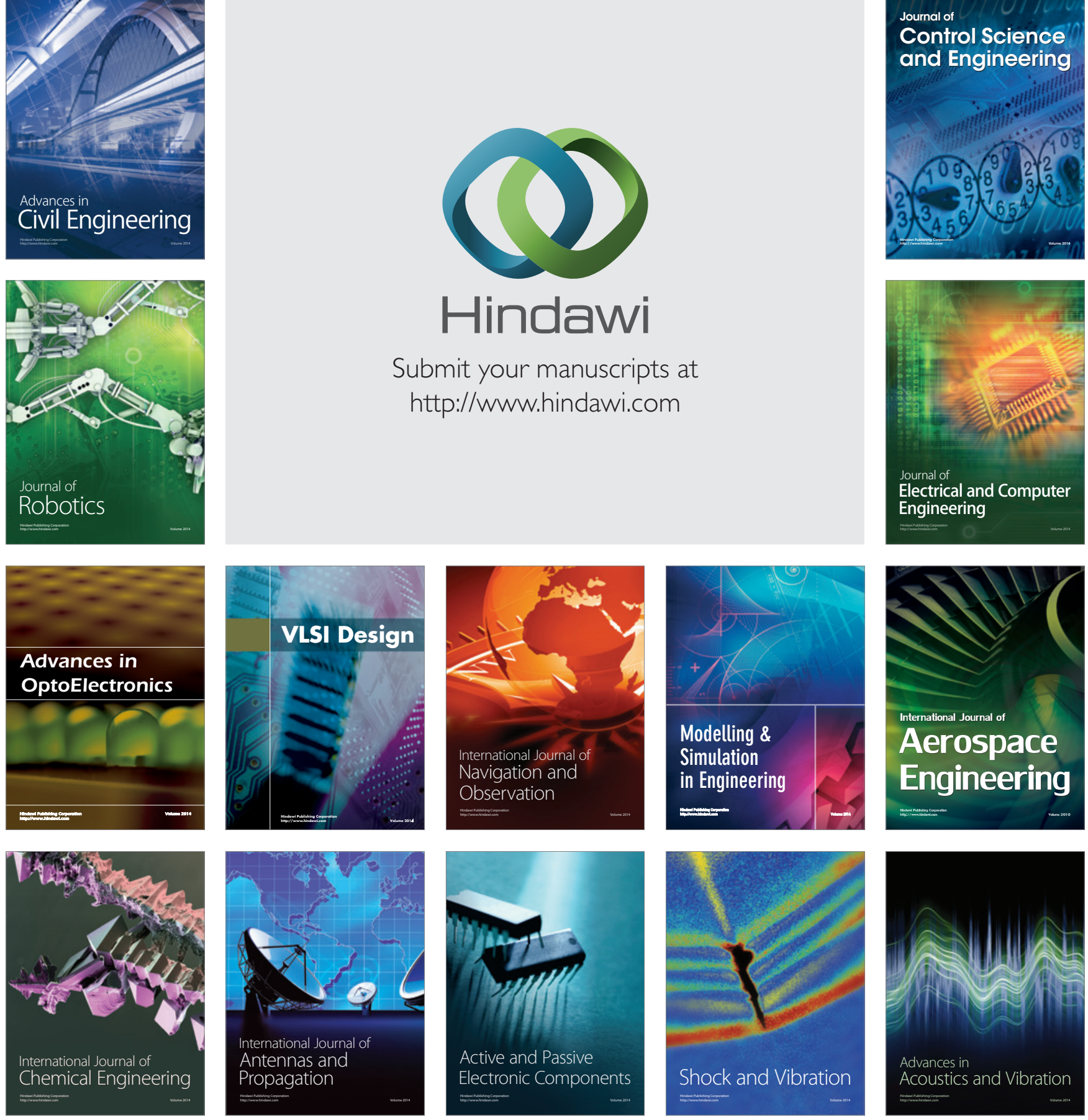